TRANSACTIONS OF THE

AMERICAN MATHEMATICAL SOCIETY

Volume 00, Number 0, Xxxx XXXX, Pages 4669-4683

S 0002-9947(97)01832-1

\title{
CONTRACTIONS ON A MANIFOLD POLARIZED BY AN AMPLE VECTOR BUNDLE
}

\author{
MARCO ANDREATTA AND MASSIMILIANO MELLA
}

\begin{abstract}
A complex manifold $X$ of dimension $n$ together with an ample vector bundle $E$ on it will be called a generalized polarized variety. The adjoint bundle of the pair $(X, E)$ is the line bundle $K_{X}+\operatorname{det}(E)$. We study the positivity (the nefness or ampleness) of the adjoint bundle in the case $r:=$ $\operatorname{rank}(E)=(n-2)$. If $r \geq(n-1)$ this was previously done in a series of papers by Ye and Zhang, by Fujita, and by Andreatta, Ballico and Wisniewski.

If $K_{X}+\operatorname{det} E$ is nef then, by the Kawamata-Shokurov base point free theorem, it supports a contraction; i.e. a map $\pi: X \longrightarrow W$ from $X$ onto a normal projective variety $W$ with connected fiber and such that $K_{X}+\operatorname{det}(E)=\pi^{*} H$, for some ample line bundle $H$ on $W$. We describe those contractions for which $\operatorname{dim} F \leq(r-1)$. We extend this result to the case in which $X$ has log terminal singularities. In particular this gives Mukai's conjecture 1 for singular varieties. We consider also the case in which $\operatorname{dim} F=r$ for every fiber and $\pi$ is birational.
\end{abstract}

\section{INTRODUCTION}

An algebraic variety $X$ of dimension $n$ (over the complex field) together with an ample vector bundle $E$ on it will be called a generalized polarized variety. The adjoint bundle of the pair $(X, E)$ is the line bundle $K_{X}+\operatorname{det}(E)$. Problems concerning adjoint bundles have drawn a lot of attention from algebraic geometers: the classical case is when $E$ is a (direct sum of) line bundles (a polarized variety), while the generalized case was motivated by the solution of the Hartshorne-Frankel conjecture by Mori $([\mathrm{Mo}])$, and by consequent conjectures of Mukai $([\mathrm{Mu}])$.

A first point of view is to study the positivity (the nefness or ampleness) of the adjoint line bundle in the case when $r=\operatorname{rank}(E)$ is about $n=\operatorname{dim} X$. This was done in a sequel of papers for $r \geq n-1$ and for a smooth manifold $X$ ([YZ], [Fu2], [ABW2]). In this paper we want to discuss the next case, namely when $\operatorname{rank}(E)=n-2$, with $X$ smooth; we obtain a complete answer which is described in the theorem (5.1). This is divided into three cases, namely when $K_{X}+\operatorname{det}(E)$ is not nef, when it is nef and not big, and finally when it is nef and big but not ample. If $n=3$ a complete picture is already contained in the famous paper of Mori ([Mo1]), while the particular case in which $E=\oplus^{n-2}(L)$ with $L$ a line bundle was also studied ([Fu1], [So]; in the singular case see [An]). Part 1 of the theorem was proved (in a slightly weaker form) by Zhang $([\mathrm{Zh}])$ and, in the case $E$ is spanned by global sections, by Wisniewski ([Wi2]).

Received by the editors March 11, 1996.

1991 Mathematics Subject Classification. Primary 14E30, 14J40; Secondary 14C20,14J45.

Key words and phrases. Vector bundle, contraction, extremal ray.

(c)1997 American Mathematical Society 
Another point of view can be the following: let $(X, E)$ be a generalized polarized variety with $X$ smooth and $\operatorname{rank} E=r$. If $K_{X}+\operatorname{det}(E)$ is nef, then by the Kawamata-Shokurov base point free theorem it supports a contraction (see Theorem 1.2); i.e. there exists a map $\pi: X \rightarrow W$ from $X$ onto a normal projective variety $W$ with connected fiber and such that $K_{X}+\operatorname{det}(E)=\pi^{*} H$ for some ample line bundle $H$ on $W$. It is not difficult to see that, for every fiber $F$ of $\pi$, we have $\operatorname{dim} F \geq(r-1)$; equality holds only if $\operatorname{dim} X>\operatorname{dim} W$. In the paper we study the "border" cases: we assume that $\operatorname{dim} F=(r-1)$ for every fiber and we prove that $X$ has a $\mathbf{P}^{r}$-bundle structure given by $\pi$ (Theorem 3.2). We consider also the case in which $\operatorname{dim} F=r$ for every fiber and $\pi$ is birational, proving that $W$ is smooth and that $\pi$ is a blow-up of a smooth subvariety (Theorem 3.1). This point of view was discussed in the case $E=\oplus^{r} L$ in the paper [AW].

Finally in section 4 we extend the Theorem 3.2 to the singular case, namely for a projective variety $X$ with log-terminal singularities. In particular this gives Mukai's conjecture 1 for singular varieties.

During the preparation of this paper we were partially supported by the MURST and GNSAGA. We would like to thank also the Max-Planck-Institut für Mathematik in Bonn and Warwick University for support and hospitality.

\section{Notations AND GENERALities}

(1.1) We use the standard notation from algebraic geometry. In particular it is compatible with that of $[\mathrm{KMM}]$, to which we refer constantly. We just explain some special definitions and propositions used frequently.

In particular, in this paper $X$ will always stand for a smooth complex projective variety of dimension $n$. Let $\operatorname{Div}(X)$ be the group of Cartier divisors on $X$; denote by $K_{X}$ the canonical divisor of $X$, an element of $\operatorname{Div}(X)$ such that $\mathcal{O}_{X}\left(K_{X}\right)=$ $\Omega_{X}^{n}$. Let $N_{1}(X)=\frac{\{1-\text { cycles }\}}{\equiv} \otimes \mathbf{R}, N^{1}(X)=\frac{\{\text { divisors }\}}{\equiv} \otimes \mathbf{R}$ and $\overline{\langle N E(X)\rangle}=$ $\overline{\text { \{effective 1-cycles }\}}$; the last is a closed cone in $N_{1}(X)$. Let $\rho(X)=\operatorname{dim}_{\mathbf{R}} N^{1}(X)$.

Suppose that $K_{X}$ is not nef; that is, there exists an effective curve $C$ such that $K_{X} \cdot C<0$.

Theorem 1.2. [KMM] Let $X$ be as above and $H$ a nef Cartier divisor such that $F:=H^{\perp} \cap \overline{\langle N E(X)\rangle} \backslash\{0\}$ is entirely contained in the set $\left\{Z \in N_{1}(X): K_{X} \cdot Z<0\right\}$, where $H^{\perp}=\{Z: H \cdot Z=0\}$. Then there exists a projective morphism $\varphi: X \rightarrow W$ from $X$ onto a normal variety $W$ with the following properties:

i) For an irreducible curve $C$ in $X, \varphi(C)$ is a point if and only if $H \cdot C=0$, if and only if $\operatorname{cl}(C) \in F$.

ii) $\varphi$ has only connected fibers.

iii) $H=\varphi^{*}(A)$ for some ample divisor $A$ on $W$.

iv) The image $\varphi^{*}: \operatorname{Pic}(W) \rightarrow \operatorname{Pic}(X)$ coincides with $\{D \in \operatorname{Pic}(X): D \cdot C=$ 0 for all $C \in F\}$.

Definition 1.3. ([KMM], definition 3-2-3). Using the notation of the above theorem, the map $\varphi$ is called a contraction (or an extremal contraction); the set $F$ is an extremal face, while the Cartier divisor $H$ is a supporting divisor for the map $\varphi$ (or the face $F$ ). If $\operatorname{dim}_{\mathbf{R}} F=1$ the face $F$ is called an extremal ray, while $\varphi$ is called an elementary contraction. 
Remark 1.4. We have also ([Mo1]) that if $X$ has an extremal ray $R$ then there exists a rational curve $C$ on $X$ such that $0<-K_{X} \cdot C \leq n+1$ and $R=R[C]:=$ $\left\{D \in\langle N E(X)\rangle: D \equiv \lambda C, \lambda \in \mathbf{R}^{+}\right\}$. Such a curve is called an extremal curve.

Remark 1.5. Let $\pi: X \rightarrow V$ denote a contraction of an extremal face $F$, supported by $H=\pi^{*} A$. Let $R$ be an extremal ray in $F$ and $\rho: X \rightarrow W$ the contraction of $R$. Then $\pi$ factors trough $\rho$ (this is because $\pi^{*} A \cdot R=0$ ).

Definition 1.6. To an extremal ray $R$ we can associate:

i) its length $l(R):=\min \left\{-K_{X} \cdot C\right.$; for $C$ a rational curve and $\left.C \in R\right\}$

ii) the locus $E(R):=$ the locus of the curves whose numerical classes are in $R\} \subset X$.

A rational curve $C$ in $R$ such that $-K_{X} \cdot C=l(R)$ will be called a minimal curve

It is usual to divide the elementary contractions associated to an extremal ray $R$ into three types, according to the dimension of $E(R)$ as follows.

Definition 1.7. We say that $\varphi$ is of fiber type, respectively divisorial type, resp. flipping type, if $\operatorname{dim} E(R)=n$, resp. $n-1$, resp. $<n-1$. Moreover an extremal ray is said to be not nef if there exists an effective $D \in \operatorname{Div}(X)$ such that $D \cdot C<0$.

The following very useful inequality was proved in [Io] and [Wi3].

Proposition 1.8. Let $\varphi$ be the contraction of an extremal ray $R, E^{\prime}(R)$ any irreducible component of the exceptional locus and $d$ the dimension of a fiber of the contraction restricted to $E^{\prime}(R)$. Then

$$
\operatorname{dim} E^{\prime}(R)+d \geq n+l(R)-1 .
$$

(1.9) Actually it is very useful to understand when a contraction is elementary, or in other words when the loci of two distinct extremal rays are disjoint. For this we will use in this paper the following results.

Proposition 1.10. [BS, Corollary 0.6.1] Let $R_{1}$ and $R_{2}$ two distinct not nef extremal rays such that $l\left(R_{1}\right)+l\left(R_{2}\right)>n$. Then $E\left(R_{1}\right)$ and $E\left(R_{2}\right)$ are disjoint.

Something can be said also if $l\left(R_{1}\right)+l\left(R_{2}\right)=n$ :

Proposition 1.11. [Fu3, Theorem 2.4] Let $\pi: X \rightarrow V$ be a birational contraction of a face $F$; suppose $n \geq 4$ and $l\left(R_{i}\right) \geq n-2$, for $R_{i}$ extremal rays in $F$. Then the exceptional loci corresponding to different extremal rays are disjoint.

Proposition 1.12. [ABW1] Let $\pi: X \rightarrow W$ be a contraction of a face such that $\operatorname{dim} X>\operatorname{dim} W$. Suppose that for every rational curve $C$ in a general fiber of $\pi$ we have $-K_{X} \cdot C \geq(n+1) / 2$. Then $\pi$ is an elementary contraction except if

a) $-K_{X} \cdot C=(n+2) / 2$ for some rational curve $C$ on $X, W$ is a point, $X$ is a Fano manifold of pseudoindex $(n+2) / 2$ and $\rho(X)=2$; and if

b) $-K_{X} \cdot C=(n+1) / 2$ for some rational curve $C$, and $\operatorname{dim} W \leq 1$.

Finally, the following definitions are used in the main theorem in section 5:

Definition 1.13. Let $L$ be an ample line bundle on $X$. The pair $(X, L)$ is called a scroll (respectively a quadric fibration, respectively a del Pezzo fibration) over a normal variety $Y$ of dimension $m$ if there exists a surjective morphism with connected fibers $\phi: X \rightarrow Y$ such that

$$
K_{X}+(n-m+1) L \approx p^{*} \mathcal{L}
$$


(respectively $K_{X}+(n-m) L \approx p^{*} \mathcal{L}$, respectively $\left.K_{X}+(n-m-1) L \approx p^{*} \mathcal{L}\right)$ for some ample line bundle $\mathcal{L}$ on $Y . X$ is called a classical scroll (respectively quadric bundle) over a projective variety $Y$ of dimension $r$ if there exists a surjective morphism $\phi: X \rightarrow Y$ such that every fiber is isomorphic to $\mathbf{P}^{n-r}$ (respectively to a quadric in $\mathbf{P}^{n-r+1}$ ) and if there exists a vector bundle $E$ of rank $n-r+1$ (respectively of rank $n-r+2$ ) on $Y$ such that $X \simeq \mathbf{P}(E)$ (respectively exists an embedding of $X$ as a subvariety of $\mathbf{P}(E)$ ).

\section{A teChNicAl CONSTRUCtion}

Let $E$ be a vector bundle of $\operatorname{rank} r$ on $X$ and assume that $E$ is ample (in Hartshorne's sense).

Remark 2.1. Let $f: \mathbf{P}^{1} \rightarrow X$ be a non-constant map, and $C=f\left(\mathbf{P}^{1}\right)$. Then $\operatorname{det} E \cdot C \geq r$.

In particular, if there exists a curve $C$ such that $\left(K_{X}+\operatorname{det} E\right) \cdot C \leq 0$ (for instance if $\left(K_{X}+\operatorname{det} E\right)$ is not nef), then there exists an extremal ray $R$ such that $l(R) \geq r$.

(2.2) Let $Y=\mathbf{P}(E)$ be the associated projective space bundle, $p: Y \rightarrow X$ the natural map onto $X$ and $\xi_{E}$ the tautological bundle of $Y$. Then we have the formula for the canonical bundle $K_{Y}=p^{*}\left(K_{X}+\operatorname{det} E\right)-r \xi_{E}$. Note that $p$ is an elementary contraction.

Assume that $K_{X}+\operatorname{det} E$ is nef but not ample, and that it is the supporting divisor of an elementary contraction $\pi: X \rightarrow W$; let $R$ be the associated extremal ray. Then $\rho(Y / W)=2$ and $-K_{Y}$ is $\pi \circ p$-ample. By the relative Mori theory over $W$ we have that there exists a ray on $N E(Y / W)$, say $R_{1}$, of length $\geq r$, not contracted by $p$, and a relative elementary contraction $\varphi: Y \rightarrow V$. We have thus the following commutative diagram:

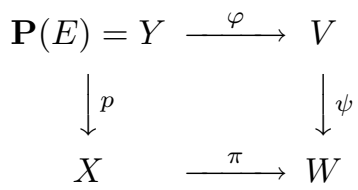

where $\varphi$ and $\psi$ are elementary contractions. Let $w \in W$ and let $F(\pi)_{w}$ be an irreducible component of $\pi^{-1}(w)$; choose also $v$ in $\psi^{-1}(w)$ and let $F(\varphi)_{v}$ be an irreducible component of $\varphi^{-1}(v)$ such that $p\left(F(\varphi)_{v}\right) \cap F(\pi)_{w} \neq \emptyset$; then, by the commutativity of the diagram, $p\left(F(\varphi)_{v}\right) \subset F(\pi)_{w}$. Since $p$ and $\varphi$ are elementary contractions of different extremal rays, we have that $\operatorname{dim}(F(\varphi) \cap F(p))=0$; that is, a curve which is contracted by $\varphi$ cannot be contracted by $p$.

In particular this implies that $\operatorname{dim}\left(p\left(F(\varphi)_{v}\right)\right)=\operatorname{dim} F(\varphi)_{v}$; therefore

$$
\operatorname{dim} F(\varphi)_{v} \leq \operatorname{dim} F(\pi)_{w}
$$

Remark 2.3. If $\operatorname{dim} F(\varphi)_{v}=\operatorname{dim} F(\pi)_{w}$. Then $\operatorname{dim} F(\psi)_{w}:=\operatorname{dim}\left(\psi^{-1}(w)\right)=r-1$; if this holds for every $w \in W$ then $\psi$ is equidimensional.

Proof. Let $Y_{w}$ be an irreducible component of $p^{-1} \pi^{-1}(w)$ such that $\varphi\left(Y_{w}\right)=$ $F(\psi)_{w}$. Then $\operatorname{dim} F(\psi)_{w}=\operatorname{dim} Y_{w}-\operatorname{dim} F(\varphi)_{v}=\operatorname{dim} Y_{w}-\operatorname{dim} F(\pi)_{w}=\operatorname{dim} F(p)=$ $r-1$.

(2.4) Slicing techniques. Let $H=\varphi^{*}(A)$ be a supporting divisor for $\varphi$ such that the linear system $|H|$ is base point free. We assume as in $(2.2)$ that $\left(K_{X}+\operatorname{det} E\right)$ 
is nef, and we refer to the diagram (2.1). The divisor $K_{Y}+r \xi_{E}=p^{*}\left(K_{X}+\operatorname{det} E\right)$ is nef on $Y$, and therefore $m\left(K_{Y}+r \xi_{E}+a H\right)$, for $m \gg 0, a \in \mathbf{N}$, is also a good supporting divisor for $\varphi$. Let $Z$ be a smooth $n$-fold obtained by intersecting $r-1$ general divisors from the linear system $|H|$, i.e. $Z=H_{1} \cap \cdots \cap H_{r-1}$ (this is what we call a slicing); let $H_{i}=\varphi^{-1} A_{i}$.

Note that the map $\varphi^{\prime}=\varphi_{\mid Z}$ is supported by $\left|m\left(K_{Y}+r \xi_{E}+a \varphi^{*} A\right)_{\mid Z}\right|$; hence, by adjunction, it is supported by $K_{Z}+r L$, where $L=\xi_{E \mid Z}$. Let $p^{\prime}=p_{\mid Z}$; by construction $p^{\prime}$ is finite.

If $T$ is (the normalization of) $\varphi(Z)$ and $\psi^{\prime}: T \rightarrow W$ is the map obtained by restricting $\psi$, then we have from (2.1) the following diagram:

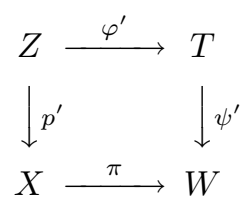

In general the map $\varphi^{\prime}$ is well understood (for instance, in the case $r=n-2$ see the results in [Fu1] or in [An]). The goal is to "transfer" the information that we have on $\varphi^{\prime}$ to the map $\pi$. The following Proposition is an example.

We refer to the diagrams and notations of the above sections; in particular $\pi: X \rightarrow W$ is the elementary contraction of the ray $R$ supported by $K_{X}+\operatorname{det} E$. Therefore $l(R) \geq r$, and by Proposition 1.8 we have

$$
\operatorname{dim} E^{\prime}(R)+d \geq n+r-1
$$

where $E^{\prime}(R)$ is an irreducible component of the exceptional locus and $d=\operatorname{dim} F(\pi)$.

Proposition 2.5. Assume that for every non-trivial fiber we have $\operatorname{dim} F(\varphi)=$ $\operatorname{dim} F(\pi)=k$. Assume also that $l(R)=r$ and that for all fibers of $\varphi$

$$
\left(F(\varphi), \xi_{E \mid F(\varphi)}\right) \simeq\left(\mathbf{P}^{k}, \mathcal{O}(1)\right) .
$$

Then $W$ has the same singularities as $T$.

Remark 2.6. The above proposition was proved in the case in which $\varphi$ is birational and $k=r$ in $[\mathrm{ABW} 2]$.

Proof. Let $w \in W$; by hypothesis and by Remark 2.3 any irreducible component $F_{i}$ of a fiber $F(\psi)_{w}$ is of dimension $r-1$. This implies also that $F_{i}=\varphi(F(p))$ for some fiber of $p$.

Lemma 2.7. There exists a fiber $F(p)_{x}$ such that $\varphi_{\mid F(p)_{x}}: F(p)_{x} \rightarrow F_{i}$ is of degree 1; that is, $\varphi_{\mid F(p)_{x}}$ is set-theoretically birational.

Proof. For every $v \in V$ we have that $\varphi_{\mid F(p)_{x}}^{-1}(v)=F(p)_{x} \cap F(\varphi)_{v}$; therefore the lemma follows if we can prove that, for a general $v \in V$, with $\psi(v)=w, p_{\mid F(\varphi)_{v}}$ : $F(\varphi)_{v} \rightarrow F(\pi)_{w}$ is set-theoretically birational.

We will need the following claim.

Claim 2.8. Let $l$ be a line in $F(\varphi) \simeq \mathbf{P}^{k}$; then $-p^{*} K_{X} \cdot l=r$.

Proof of the claim. Let $C$ a minimal curve in the ray $R$ (see Definition 1.6); let $\nu: \mathbf{P}^{1} \rightarrow C$ be its normalization. Thus $\nu^{*} E_{\mid C}=\oplus^{r} \mathcal{O}(1)$, and therefore $Y_{C}=$ $\mathbf{P}\left(\nu^{*} E_{C}\right)=\mathbf{P}^{1} \times \mathbf{P}^{r-1}$. Let $\tilde{\nu}: Y_{C} \rightarrow Y$ be the map induced by $\nu$ and let $\tilde{l}$ be a section of $\sigma: Y_{C} \rightarrow \mathbf{P}^{1}$; note that $\nu \sigma: \tilde{l} \rightarrow C$ is birational. Note also that $\tilde{\nu}^{*} \xi_{E}$ is 
the tautological bundle for $Y_{C}$; thus $1=\tilde{\nu}^{*} \xi_{E} \cdot \tilde{l}=\xi_{E} \cdot \tilde{\nu}_{*} \tilde{l}$, hence $\tilde{\nu}_{*} \tilde{l}=l$. Therefore $p_{*} l=C$ and $-p^{*} K_{X} \cdot l=-K_{X} \cdot p_{*} l=-K_{X} \cdot C=r$.

Let $R$ be the ramification divisor of $p^{\prime}: Z \rightarrow X$ defined by the formula

$$
K_{Z}=p^{*} K_{X}+R
$$

Let $l$ be a line in $F(\varphi)=\mathbf{P}^{k} \subset Z$; on one side we have that $-K_{Z} \cdot l=r$; on the other, by the above claim, $p^{*} K_{X} \cdot l=r$. Therefore $R \cdot l=0$. Thus either $F(\varphi) \subset R$ or $F(\varphi) \cap R=\emptyset$. We want to prove that the latter is the case.

Lemma 2.9. For a general choice of $Z$ the ramification divisor $R$ does not contain $F(\varphi)=\mathbf{P}^{k} \subset Z$; therefore $F(\varphi) \cap R=\emptyset$.

Proof. It is enough to prove that there exists an $x \in F(\pi)_{w}$ such that $p^{-1}(x) \cap Z$ consists of $d$ distinct points, where $d=\operatorname{deg}\left(p^{\prime}: Z \rightarrow X\right)$. Observe that this is true for every $x_{1} \in X$ outside the branch locus and $d=\varphi^{*} A^{r-1} \cdot F(p)_{x_{1}}=$ $\varphi^{*} A^{r-1} \cdot F(p)_{x}$, where $Z=\varphi^{*} A_{1} \cap \ldots \cap \varphi^{*} A_{r-1}$ and $A_{i} \in|A|$. Moreover $p^{-1}(x) \cap$ $Z=\bigcup_{i} p^{-1}(x) \cap F(\varphi)_{v_{i}}$, where the union is taken over all $v_{i} \in T \cap F_{i}$. Since $\varphi_{\mid F(p)_{x}}: F(p)_{x} \rightarrow F_{i}$ is generically unramified, choosing generic sections $A_{i} \in|A|$ yields that $p^{-1}(x) \cap F(\varphi)_{v_{i}}$ is a reduced cycle of length $d_{i}$ for any $i$ and $\sum_{i} d_{i}=d$. Hence $F(\varphi) \cap R=\emptyset$.

The exact sequence

$$
\mathcal{I} / \mathcal{I}^{2} \rightarrow \Omega_{Z / X} \otimes \mathcal{O}_{F(\varphi)} \rightarrow \Omega_{F(\varphi) / X} \rightarrow 0
$$

yields that also $p_{\mid F(\varphi)}: \mathbf{P}^{k} \rightarrow F(\pi)$ is unramified. Let $f: \tilde{F} \rightarrow F(\pi)$ be the normalization and $g: \mathbf{P}^{k} \rightarrow \tilde{F}$ the map induced by $p$; then $g$ is unramified and $\tilde{F}$ is smooth by Zariski's Main Theorem. Therefore $\tilde{F} \simeq \mathbf{P}^{k}$ by Lazarsfeld's result and $g$ is an isomorphism; thus $p_{\mid F(\varphi)}$ is of degree 1 .

Let $\varphi_{\mid F(p)}: F(p) \rightarrow F_{i}$ be as in the lemma, that is, $\varphi_{\mid F(p)}$ is set-theoretically birational. Let us follow an argument as in [Fu1, Lemma 2.12]. We can assume that the divisor $A$ is very ample. Using Bertini's theorem we choose $r-1$ divisors $A_{i} \in|A|$ as above such that, if $T=\bigcap_{i} A_{i}$, then $T \cap \psi^{-1}(w)_{\text {red }}=N$ is a reduced 0 -cycle and $Z=H_{1} \cap \cdots \cap H_{r-1}$ is a smooth $n$-fold, where $H_{i}=\varphi^{-1} A_{i}$. Moreover the number of points in $N$ is given by $A^{r-1} \cdot \psi^{-1}(w)_{r e d}=\sum_{i} A^{r-1} \cdot F_{i}=\sum_{i} d_{i}$. Note that, by the projection formula, we have $A^{r-1} \cdot F_{i}=\varphi^{*} A^{r-1} \cdot F(p)$; here we use the fact that the map $\varphi_{\mid F(p)}$ is set-theoretically birational. Moreover, since $p$ is a projective bundle, the last number is constant, i.e. $\varphi^{*} A^{r-1} \cdot F(p)=d$ for all fibers $F(p)$; that is, the $d_{i}$ 's are constant.

Using that $\psi^{\prime}:=\psi_{\mid T}: T \rightarrow W$ is proper and finite over $w$, we now take a small enough neighborhood $U$ of $w$, in the metric topology, such that any connected component $U_{\lambda}$ of $\psi^{-1}(U) \cap T$ meets $\psi^{-1}(w)$ in a single point. Let $\psi_{\lambda}$ be the restriction of $\psi$ to $U_{\lambda}$ and $m_{\lambda}$ its degree. Then $\operatorname{deg} \psi^{\prime}=\sum m_{\lambda} \geq \sum_{i} d_{i}=\sum_{i} d$, and equality holds if and only if $\psi$ is not ramified at $w$ (remember that $\sum_{i} d_{i}$ is the number of $\left.U_{\lambda}\right)$.

The generic $F(\psi)_{w}$ is irreducible and generically reduced. Note that we can choose $\tilde{w} \in W$ such that $\psi^{-1}(\tilde{w})=\varphi(F(p))$ and $\operatorname{deg} \psi^{\prime}=A^{r-1} \cdot \psi^{-1}(\tilde{w})$; the latter is possible by the choice of generic sections of $|A|$. Hence, by the projection formula, $\operatorname{deg} \psi^{\prime}=A^{r-1} \cdot \psi^{-1}(\tilde{w})=\varphi^{*} A^{r-1} \cdot F(p)=d$; that is, $m_{\lambda}=1$, and the fibers are irreducible. Since $W$ is normal we can conclude, by Zariski's Main Theorem, that $W$ has the same singularities as $T$. 
Corollary 2.10. In the hypothesis of the above proposition assume also that either $\varphi$ is birational and $k=r$, or that $\varphi$ is of fiber type and $k=(r-1)$. Then $W$ is smooth.

Proof. [AW, Theorem 4.1] applies to the map $\varphi$ and gives that $T$ is smooth and $\varphi$ satisfies the hypothesis of Proposition 2.5 (for the fiber type case it is actually a theorem in [Fu1]). Thus by Proposition 2.5 also $W$ is smooth.

\section{Some General applications}

As an application of the above construction we will prove the following proposition; the case $r=n-1$ was proved in [ABW2].

Theorem 3.1. Let $X$ be a smooth projective complex variety and $E$ be an ample vector bundle of rank $r$ on $X$. Assume that $K_{X}+\operatorname{det} E$ is nef and big but not ample, and let $\pi: X \rightarrow W$ be the contraction supported by $K_{X}+\operatorname{det} E$. Assume also that $\pi$ is a divisorial elementary contraction, with exceptional divisor $D$, and that $\operatorname{dim} F \leq r$ for all fibers $F$. Then $W$ is smooth, $\pi$ is the blow-up of a smooth subvariety $B:=\pi(D)$, and $E=\pi^{*} E^{\prime} \otimes[-D]$, for some ample $E^{\prime}$ on $W$.

Proof. In the previous section (2.10) we have proved that $W$ is smooth. Therefore $\pi$ is a birational morphism between smooth varieties with exceptional locus a prime divisor and with equidimensional non-trivial fibers; by [AW, Corollary 4.11] this implies that $\pi$ is a blow-up of a smooth subvariety in $W$.

We want to show that $E=\pi^{*} E^{\prime} \otimes[-D]$. Let $D_{1}$ be the exceptional divisor of $\varphi$; first we claim that $\xi_{E}+D_{1}$ is a good supporting divisor for $\varphi$. Let $C_{1}$ be a minimal curve in the ray $R_{1}$ (see Definition 1.6), contracted by $\varphi$; we have that $\xi_{E} \cdot C_{1}=1$. Observe that $\left(\xi_{E}+D_{1}\right) \cdot C_{1}=0$, while $\left(\xi_{E}+D_{1}\right) \cdot C>0$ for any curve $C$ with $\varphi(C) \neq p t$ (in fact, $\xi_{E}$ is ample and $D_{1} \cdot C \geq 0$ for such a curve). Thus $\xi_{E}+D_{1}=\varphi^{*} A$ for some ample $A \in \operatorname{Pic}(V)$; moreover, using the projection formula, $A \cdot l=1$, for any line $l$ in the fiber of $\psi$. Hence, by Grauert's theorem, $V=\mathbf{P}\left(E^{\prime}\right)$ for some ample vector bundle $E^{\prime}$ on $W$. This yields, by the commutativity of diagram (1), $E \otimes D=p_{*}\left(\xi_{E}+D_{1}\right)=p_{*} \varphi^{*} A=\pi^{*} \psi_{*} A=\pi^{*} E^{\prime}$.

Similarly, for the fiber type case, we have the following.

Theorem 3.2. Let $X$ be a smooth projective complex variety and $E$ be an ample vector bundle of rank $r$ on $X$. Assume that $K_{X}+\operatorname{det} E$ is nef and let $\pi: X \rightarrow W$ be the contraction supported by $K_{X}+\operatorname{det} E$. Assume that $r \geq(n+1) / 2$ and $\operatorname{dim} F \leq$ $r-1$ for any fiber $F$ of $\pi$. Then $\pi$ is a fiber type contraction, $W$ is smooth, and for any fiber $F \simeq \mathbf{P}^{r-1}$ and $E_{\mid F}=\oplus^{r} \mathcal{O}(1)$.

Proof. Note that, by Proposition 1.8, $\pi$ is a contraction of fiber type and all the fibers have dimension $r-1$. Moreover the contraction is elementary, by Proposition 1.12 .

By Corollary $2.10 \mathrm{~W}$ is smooth. We want to use an inductive argument to prove the theorem. If $\operatorname{dim} W=0$ then this is Mukai's conjecture 1, which was proved by Peternell, Kollár, and Ye and Zhang (see for instance [YZ]). Let the theorem be true for dimension $m-1$. Note that the locus over which the fiber is not $\mathbf{P}^{r-1}$ is discrete. In fact take a general hyperplane section $A$ of $W$, and $X^{\prime}=\pi^{-1}(A)$; then $\pi_{\mid X^{\prime}}: X^{\prime} \rightarrow A$ is again a contraction supported by $K_{X^{\prime}}+\operatorname{det} E_{\mid X^{\prime}}$, such that $r \geq((n-1)+1) / 2$. Thus by induction $A$ is smooth and all fibers over $A$ are $\mathbf{P}^{r-1}$. 
Let $U$ be an open disk in the complex topology such that $U \cap \operatorname{Sing} W=\{0\}$. Then by Lemma 3.3, below, we obtain locally, in the complex topology, a $\pi$-ample line bundle $L$ that restricted to the general fiber is $\mathcal{O}(1)$. Thus, as in [Fu1, Prop. 2.12 ], we can prove that all the fibers are $\mathbf{P}^{r-1}$.

Lemma 3.3. Let $X$ be a complex manifold and $(W, 0)$ an analytic germ such that $W \backslash\{0\} \simeq \Delta^{m} \backslash\{0\}$. Assume we have a holomorphic map $\pi: X \rightarrow W$ with $-K_{X}$ $\pi$-ample; assume also that $F \simeq \mathbf{P}^{r}$ for all fibers of $\pi, F \neq F_{0}=\pi^{-1}(0)$, and that codim $F_{0} \geq 2$. Then there exists a line bundle $L$ on $X$ such that $L$ is $\pi$-ample and $L_{\mid F}=\mathcal{O}(1)$.

Proof. (see also $\left[\mathrm{ABW} 2\right.$, pp. 338-339]) Let $W^{*}=W \backslash\{0\}$ and $X^{*}=X \backslash F_{0}$. By abuse of notation set $\pi=\pi_{\mid X^{*}}: X^{*} \rightarrow W^{*}$; it follows immediately that $R^{1} \pi_{*} \mathbf{Z}_{X^{*}}=$ 0 and $R^{2} \pi_{*} \mathbf{Z}_{X^{*}}=\mathbf{Z}$.

Using the Leray spectral sequence, we have that

$$
E_{2}^{0,2}=\mathbf{Z} \text { and } E_{2}^{p, 1}=0 \text { for any } p .
$$

Therefore $d_{2}: E_{2}^{0,2} \rightarrow E_{2}^{2,1}$ is the zero map, and moreover we have the following exact sequence:

$$
0 \rightarrow E_{\infty}^{0,2} \rightarrow E_{2}^{0,2} \stackrel{d_{3}}{\rightarrow} E_{2}^{3,0},
$$

since the only non-zero map from $E_{2}^{0,2}$ is $d_{3}$ and hence $E_{\infty}^{0,2}=k e r d_{3}$. On the other hand we have also, in a natural way, a surjective map $H^{2}\left(X^{*}, \mathbf{Z}\right) \rightarrow E_{\infty}^{0,2} \rightarrow 0$. Thus we get the following exact sequence:

$$
H^{2}\left(X^{*}, \mathbf{Z}\right) \stackrel{\alpha}{\rightarrow} E_{2}^{0,2} \rightarrow E_{2}^{3,0}=H^{3}\left(W^{*}, \mathbf{Z}\right) .
$$

We want to show that $\alpha$ is surjective. If $\operatorname{dim} W:=w \geq 3$ then $H^{3}\left(W^{*}, \mathbf{Z}\right)=0$ and we are done. Suppose $w=2$; then $H^{3}\left(W^{*}, \mathbf{Z}\right)=\mathbf{Z}$; note that the restriction of $-K_{X}$ gives a non-zero class (in fact it is $r+1$ times the generator) in $E_{2}^{0,2}$ and is mapped to zero in $E_{2}^{0,3}$; thus the mapping $E_{2}^{0,2} \rightarrow E_{2}^{3,0}$ is the zero map and $\alpha$ is surjective. Since $F_{0}$ is of codimension at least 2 in $X$, the restriction map $H^{2}(X, \mathbf{Z}) \rightarrow H^{2}\left(X^{*}, \mathbf{Z}\right)$ is a bijection. By the vanishing of $R_{i} \pi_{*} \mathcal{O}_{X}$ we get $H^{2}\left(X, \mathcal{O}_{X}\right)=H^{2}\left(W, \mathcal{O}_{W}\right)=0$; hence also $\operatorname{Pic}(X) \rightarrow H^{2}(X, \mathbf{Z})$ is surjective. Let $L \in \operatorname{Pic}(X)$ be a preimage of a generator of $E_{2}^{0,2}$. By construction $L_{t}$ is $\mathcal{O}(1)$, for $t \in W^{*}$. Moreover $(r+1) L=-K_{X}$ on $X^{*}$; thus, again by the codimension of $X^{*}$, this is true on $X$ and $L$ is $\pi$-ample.

\section{An approach to the singular case}

The following theorem arose during a discussion between us and J.A. Wiśniewski; we would like to thank him. The idea to investigate this argument originated with Zhang [Zh2]. For the definition of log-terminal singularity we refer to [KMM].

Theorem 4.1. Let $X$ be an n-dimensional log-terminal projective variety and $E$ be an ample vector bundle of rank $r$. Assume that $K_{X}+\operatorname{det} E$ is nef and let $\pi: X \rightarrow W$ be the contraction supported by $K_{X}+\operatorname{det} E$. Assume also that for any fiber $F$ of $\pi$ $\operatorname{dim} F \leq r-1$, and that $r \geq(n+1) / 2$ and codimSing $(X)>\operatorname{dim} W$. Then $X$ and $W$ are smooth and, for any fiber, $F \simeq \mathbf{P}^{r-1}$. 
Proof. We will prove that $X$ is smooth. Then we can apply Theorem 3.2. We consider in this case the associated projective space bundle $Y$ and the commutative diagram

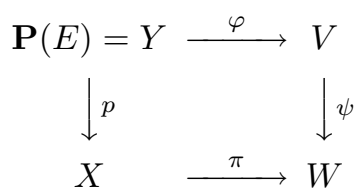

as in 2.1); it is immediate that $Y$ is Gorenstein and log-terminal; in particular it has Cohen-Macaulay singularities. Moreover, as in $(3.1) \operatorname{dim} F(\varphi) \leq \operatorname{dim} F(\pi)$ and the map $\varphi$ is supported by $K_{Y}+r H$, where $H=\xi_{E}+A$, with $\xi_{E}$ the tautological line bundle and $A$ a pull-back of an ample line bundle from $V$. It is known that a contraction supported by $K_{Y}+r H$ on a $\log$ terminal variety has to have fibers of dimension $\geq(r-1)$ and of dimension $\geq r$ in the birational case ([AW, remark 3.1.2]). Thus $\varphi$ is not birational and all fibers have dimension $r-1$; moreover, by the Kobayashi-Ochiai criterion the general fiber is $F \simeq \mathbf{P}^{r-1}$. Imitating the proof of [BS, Prop 1.4], we have only to show that there are no fibers of $\varphi$ entirely contained in $\operatorname{Sing}(Y)$. Note that, by construction, $\operatorname{Sing}(Y) \subset p^{-1}(\operatorname{Sing} X)$. Hence no fibers $F$ of $\varphi$ can be contained in $\operatorname{Sing}(Y)$, and therefore the same proof of [BS, Prop. 1.4] applies. It follows that $V$ is nonsingular, and $\varphi: Y \rightarrow V$ is a classical scroll. In particular $Y$ is nonsingular, and therefore also $X$ is nonsingular.

As a corollary we obtain Mukai's conjecture 1 in the log terminal case (see also $[\mathrm{Zh} 2])$.

Corollary 4.2. Let $X$ be an $n$-dimensional log-terminal projective variety and $E$ an ample vector bundle of rank $n+1$, such that $c_{1}(E)=c_{1}(X)$. Then $(X, E)=$ $\left(\mathbf{P}^{n}, \oplus^{n+1} \mathcal{O}_{\mathbf{P}^{n}}(1)\right)$.

\section{MAIN THEOREM}

This section is devoted to the proof of the following theorem.

Theorem 5.1. Let $X$ be a smooth projective variety over the complex field of dimension $n \geq 3$ and $E$ an ample vector bundle on $X$ of rank $r=n-2$. Then we have:

1) $K_{X}+\operatorname{det}(E)$ is nef unless $(X, E)$ is one of the following:

i) there exist a smooth $n$-fold, $W$, and a morphism $\phi: X \rightarrow W$ expressing $X$ as a blow up of a finite set $B$ of points and an ample vector bundle $E^{\prime}$ on $W$ such that $E=\phi^{*} E^{\prime} \otimes\left[-\phi^{-1}(B)\right]$.

Assume from now on that $(X, E)$ is not as in $i$ ) above (that is eventually consider the new pair $\left(W, E^{\prime}\right)$ coming from $\left.\left.i\right)\right)$.

ii) $X=\mathbf{P}^{n}$ and $E=\oplus^{n-2} \mathcal{O}(1)$ or $\oplus^{2} \mathcal{O}(2) \oplus^{n-4} \mathcal{O}(1)$ or $\mathcal{O}(2) \oplus^{n-3} \mathcal{O}(1)$ or $\mathcal{O}(3) \oplus^{n-3} \mathcal{O}(1)$.

iii) $X=\mathbf{Q}^{n}$ and $E=\oplus^{n-2} \mathcal{O}(1)$ or $\mathcal{O}(2) \oplus^{n-3} \mathcal{O}(1)$ or $\mathbf{E}(2)$ with $\mathbf{E}$ a spinor bundle on $\mathbf{Q}^{n}$.

iv) $X=\mathbf{P}^{2} \times \mathbf{P}^{2}$ and $E=\oplus^{2} \mathcal{O}(1,1)$.

v) $X$ is a del Pezzo manifold with $b_{2}=1$, i.e. $\operatorname{Pic}(X)$ is generated by an ample line bundle $\mathcal{O}(1)$ such that $\mathcal{O}(n-1)=\mathcal{O}\left(-K_{X}\right)$ and $E=$ $\oplus^{n-1} \mathcal{O}(1)$

vi) $X$ is a classical scroll or a quadric bundle over a smooth curve $Y$. 
vii) $X$ is a classical scroll over a smooth surface $Y$.

2) If $K_{X}+\operatorname{det}(E)$ is nef then it is big unless there exists a morphism $\phi: X \rightarrow W$ onto a normal variety $W$ supported by (a large multiple of) $K_{X}+\operatorname{det}(E)$ and $\operatorname{dim}(W) \leq 3$; let $F$ be a general fiber of $\phi$ and $E^{\prime}=E_{\mid F}$. We have the following according to $s=\operatorname{dim} W$ :

i) If $s=0$ then $X$ is a Fano manifold and $K_{X}+\operatorname{det}(E)=0$. If $n \geq 6$ then $b_{2}(X)=1$ except if $X=\mathbf{P}^{3} \times \mathbf{P}^{3}$ and $E=\oplus^{4} \mathcal{O}(1,1)$.

ii) If $s=1$ then $W$ is a smooth curve and $\phi$ is a flat (equidimensional) map. Then $\left(F, E^{\prime}\right)$ is one of the pair described in [PSW]; in particular, $F$ is either $\mathbf{P}^{n-1}$ or a quadric or a del Pezzo variety. If $n \geq 6$ then $\pi$ is an elementary contraction. If the general fiber is $\mathbf{P}^{n-1}$ then $X$ is a classical scroll, while if the general fiber is $\mathbf{Q}^{n-1}$ then $X$ is a quadric bundle.

iii) If $s=2$ and $n \geq 5$, then $W$ is a smooth surface, $\phi$ is a flat map and $\left(F, E^{\prime}\right)$ is one of the pair described in the Main Theorem of [Fu2]. If the general fiber is $\mathbf{P}^{n-2}$, all the fibers are $\mathbf{P}^{n-2}$.

iv) If $s=3$ and $n \geq 5$, then $W$ is a 3-fold with at most isolated singularities and $X$ has at most isolated fibers of dimension $n-2$; all fibers over smooth point are isomorphic to $\mathbf{P}^{n-3}$.

3) Assume finally that $K_{X}+\operatorname{det}(E)$ is nef and big but not ample. Then a high multiple of $K_{X}+\operatorname{det}(E)$ defines a birational map, $\varphi: X \rightarrow X^{\prime}$, which contracts an "extremal face" (see section 2). Let $R_{i}$, for $i$ in a finite set of indices, be the extremal rays spanning this face; call $\rho_{i}: X \rightarrow W$ the contraction associated to one of the $R_{i}$. Then each $\rho_{i}$ is birational and divisorial; if $D$ is one of the exceptional divisors (we drop the index) and $B=\rho(D)$ we have that $\operatorname{dim}(B) \leq 1$ and the following possibilities occur:

i) $\operatorname{dim} B=0, D=\mathbf{P}^{n-1}$ and $D_{\mid D}=\mathcal{O}(-2)$; moreover $E_{\mid D} \simeq \oplus^{n-2} \mathcal{O}(1)$.

ii) $\operatorname{dim} B=0, D$ is a (possibly singular) quadric, $\mathbf{Q}^{n-1}$, and $D_{\mid D}=\mathcal{O}(-1)$; moreover $E_{\mid D}=\oplus^{n-2} \mathcal{O}(1)$.

iii) $\operatorname{dim} B=1, W$ and $Z$ are smooth projective varieties, and $\rho$ is the blow-up of $W$ along $Z$. Moreover $E_{\mid F}=\oplus^{n-2} \mathcal{O}(1)$.

If $n>3$ then $\varphi$ is a composition of "disjoint" extremal contractions as in i), ii) or iii).

Proof of part 1) of Theorem 5.1. Let $(X, E)$ be a generalized polarized variety and assume that $K_{X}+\operatorname{det}(E)$ is not nef. Then there exist on $X$ a finite number of extremal rays, $R_{1}, \ldots, R_{s}$, such that $\left(K_{X}+\operatorname{det}(E)\right) \cdot R_{i}<0$, and therefore, by Remark 2.1, $l\left(R_{i}\right) \geq n-1$.

Consider one of this extremal rays, $R=R_{i}$, and let $\rho: X \rightarrow Y$ be its associated elementary contraction. Then $L:=-\left(K_{X}+\operatorname{det}(E)\right)$ is $\rho$-ample and so is the vector bundle $E_{1}:=E \oplus L$; moreover $K_{X}+\operatorname{det}\left(E_{1}\right)=\mathcal{O}_{X}$ relative to $\rho$. To proceed we need a relative version of the theorem in $[A B W 2]$ which studies the positivity of the adjoint bundle in the case of $\operatorname{rank} E_{1}=n-1$. More precisely, we assume not that $E_{1}$ is ample but that it is $\rho$-ample (or equivalently a local statement in a neighborhood of the exceptional locus of the extremal ray $R$ ). For this we notice that the theorem in $[A B W 2]$ is true also in the relative case and can be proved verbatim using the relative minimal model theory instead of the absolute (see [KMM]; see also section 2 of $[\mathrm{AW}]$ for a discussion of the local setup).

Assume first that $\rho$ is birational; then $K_{X}+\operatorname{det}\left(E_{1}\right)$ is $\rho$-nef and $\rho$-big; note also that, since $l\left(R_{i}\right) \geq n-1, \rho$ is divisorial. Therefore we are in the (relative) 
case $\mathrm{C}$ of the theorem in [ABW2] (see also Theorem 3.1 with $r=n-1$ ); this implies that $Y$ is smooth and $\rho$ is the blow-up of a point in $Y$. Since $l\left(R_{i}\right) \geq n-1$, the exceptional loci of the birational rays are pairwise disjoint by Proposition 1.10. This gives Theorem 5.1 (i): the birational extremal rays have disjoint exceptional loci which are divisors isomorphic to $\mathbf{P}^{n-1}$ and which contract simultaneously to smooth distinct points on a $n$-fold $W$. The description of $E$ follows trivially (see also $\lfloor\mathrm{ABW} 2])$.

If $\rho$ is not birational then we are in case B of the theorem in [ABW2]; from this we obtain similarly as above the other cases of Theorem 5.1, with some trivial computations needed to recover $E$ from $E_{1}$. Note that in the case of fibration over a surface, since all fibers are $\mathbf{P}^{n-2}$, then $n-1=l(R)>\operatorname{det} E \cdot R_{i} \geq n-2$; thus $l(R)=n-1$ and $\operatorname{det} E \cdot R_{i}=n-2$. Then $-\left(\operatorname{det} E+K_{X}\right)$ is a tautological bundle for the fibration, and the fibration is a scroll. This part was also independently proved in $[\mathrm{Ma}]$

Proof of part 2) of Theorem 5.1. Let $K_{X}+\operatorname{det} E$ be nef but not big; then it is the supporting divisor of a face $F=\left(K_{X}+\operatorname{det} E\right)^{\perp}$. Using $([\mathrm{KMM}])$ we can say that there exists a map $\pi: X \rightarrow W$ which is given by a high multiple of $K_{X}+\operatorname{det} E$ and which contracts the curves in the face. Since $K_{X}+\operatorname{det} E$ is not big, we have that $\operatorname{dim} W<\operatorname{dim} X$. Moreover for every rational curve $C$ in a general fiber of $\pi$ we have $-K_{X} \cdot C \geq n-2$ by Remark 2.1. We apply Proposition 1.12, which, together with the above inequality on $-K_{X} \cdot C$, gives that $\pi$ is an elementary contraction if $n \geq 5$ unless either $n=6, W$ is a point and $X$ is a Fano manifold of pseudoindex 4 and $\rho(X)=2$, or $n=5$ and $\operatorname{dim} W \leq 1$.

By Proposition 1.8 we have the inequality

$$
n+d \geq n+n-2-1,
$$

where $d$ is the dimension of a fiber; in particular it follows that $\operatorname{dim} W \leq 3$.

Case $5.2(\operatorname{dim} W=0)$. Then $K_{X}+\operatorname{det} E=0$, and therefore $X$ is a Fano manifold. By what just said above we have that $b_{2}(X)=1$ if $n \geq 6$, with an exception which is a particular case of the following lemma for $n=6$.

Lemma 5.3. Let $X$ be an $n$-dimensional projective manifold, $E$ an ample vector bundle on $X$ of rank $r+1$ such that $K_{X}+\operatorname{det} E=0$, and $n=2 r$. Assume moreover that $b_{2} \geq 2$. Then $X=\mathbf{P}^{r} \times \mathbf{P}^{r}$ and $E=\oplus^{r} \mathcal{O}(1,1)$.

Proof. The lemma is a slight generalization of [Wi1, Prop. B]; the proof is similar and for more details we refer to that paper. In particular, as in [Wi1] we can see that $X$ has two extremal rays whose contractions $\pi_{i}, i=1,2$, are of fiber type with equidimensional fibers onto $r$-folds $W_{i}$ and with general fiber $F_{i} \simeq \mathbf{P}^{r}$. We claim that the $W_{i}$ are smooth and thus $W_{i} \simeq \mathbf{P}^{r}$. The contractions $\pi_{i}$ are supported by $K_{X}+\operatorname{det} E_{i}^{\prime}$, with $E_{i}^{\prime}$ an ample vector bundle $\left(E_{i}^{\prime}=E \times \pi^{*} A_{i}\right.$ with $A_{i}$ ample on $W_{i}$ ). Therefore we are in the hypothesis of Proposition 3.2. Thus the $W_{i}$ are smooth and all the fibers are $\mathbf{P}^{r}$.

Let $T=\bigcap_{i=1}^{r-1} H_{i}$, where $H_{i}$ are general elements of $\pi_{1}^{*}(\mathcal{O}(1))$. We claim that $T \simeq \mathbf{P}^{1} \times \mathbf{P}^{r}$. In fact $T$ is smooth and $\pi_{1 \mid T}$ makes $T$ a projective bundle over a line (since $H^{2}\left(\mathbf{P}^{1}, \varnothing^{*}\right)=0$ ), that is, $T=\mathbf{P}(\mathcal{F})$. Moreover $\pi_{2_{\mid T}}$ is onto $\mathbf{P}^{r}$; therefore the claim follows. Therefore we conclude that $\pi_{i}^{*} \mathcal{O}_{\mathbf{P}^{r}}(1)_{\mid F_{i}} \simeq \mathcal{O}_{\mathbf{P}^{r}}(1)$ for $i=1,2$. This implies, by Grauert's Theorem, that the two fibrations are classical scrolls, that is, 
$X=\mathbf{P}\left(\mathcal{F}_{i}\right)$, for $i=1,2$; moreover, computing the canonical class of $X$, the $\mathcal{F}_{i}$ are ample and the lemma easily follows.

Case $5.4(\operatorname{dim} W=1)$. Then $W$ is a smooth curve and $\pi$ is a flat map. Let $F$ be a general fiber; then $F$ is a smooth Fano manifold and $E_{\mid F}$ is an ample vector bundle on $F$ of rank $n-2=\operatorname{dim} F-1$ such that $-K_{F}=\operatorname{det}\left(E_{\mid F}\right)$. These pairs $\left(F, E_{\mid F}\right)$ are classified in the Main Theorem of [PSW]; in particular, if $\operatorname{dim} F \geq 5, F$ is either $\mathbf{P}^{n-1}$ or $\mathbf{Q}^{n-1}$ or a del Pezzo manifold with $b_{2}(F)=1$. Moreover, if $n \geq 6$, then $\pi$ is an elementary contraction by Proposition 1.12.

Claim 5.5. Let $n \geq 6$ and assume that the general fiber is $\mathbf{P}^{n-1}$. Then $X$ is a classical scroll and $E_{\mid F}$ is the same for all $F$.

Proof. (See also [Fu2].) Let $S=W \backslash U$ be the locus of points over which the fiber is not $\mathbf{P}^{n-1}$. Over $U$ we have a projective fiber bundle. Since $H^{2}\left(U, \mathcal{O}^{*}\right)=0$ we can associate this $\mathbf{P}$-bundle to a vector bundle $\mathcal{F}$ over $U$. Let $Y=\mathbf{P}(\mathcal{F})$ and $H$ the tautological bundle; by abuse of language let $H$ be the extension of $H$ to $X$. Since $\pi$ is elementary, $H$ is an ample line bundle on $X$. Therefore by semicontinuity $\Delta\left(F, H_{F}\right) \geq \Delta\left(G, H_{G}\right)$, for any fiber $G$, where $\Delta(X, L)$ is Fujita's delta-genus. In our case this yields $0=\Delta\left(F, H_{F}\right) \geq \Delta\left(G, H_{G}\right) \geq 0$. Moreover by flatness $\left(H_{G}\right)^{n-1}=\left(H_{F}\right)^{n-1}=1$; by the Fujita classification of the pairs of delta genus zero we conclude that all $G$ are equal to $\mathbf{P}^{n-1}$. Using again the Main Theorem of [PSW], we see that $E_{\mid G}$ is decomposable, hence rigid; that is, the decomposition is the same along all fibers of $\pi$. This concludes the proof of the claim.

Claim 5.6. Let $n \geq 6$ and assume that the general fiber is $\mathbf{Q}^{n-1}$. Then $X$ is a quadric bundle.

Proof. As above, let $S=W \backslash U$ be the locus of points over which the fiber is not a smooth quadric. Let $X^{*}=\pi^{-1}(U)$; then we can embed $X^{*}$ in a fiber bundle of projective spaces over $U$, since it is locally trivial. Associate this $P$-bundle over $U$ to a projective bundle and argue as before.

Case $5.7(\operatorname{dim} W=2)$. Assume that $n \geq 5$; then $\pi$ is an elementary contraction. This implies first, by [ABW2, Prop. 1.4.1], that $W$ is smooth; secondly that $\pi$ is equidimensional, hence flat and the general fiber is $\mathbf{P}^{n-2}$ or $\mathbf{Q}^{n-2}$, see [Fu2].

Claim 5.8. Let $n \geq 5$ and let the general fiber be $\mathbf{P}^{n-2}$; then for any fiber $F \simeq \mathbf{P}^{n-2}$ and $E_{\mid F}$ is the same for all $F$.

Proof. Let $S \subset W$ be the locus of fibers that are not $\mathbf{P}^{n-2}$; then $\operatorname{dim} S \leq 0$ since $W$ is smooth. In fact, over a generic hyperplane section on $W$ all fibers are $\mathbf{P}^{n-2}$ by [ABW2]. Let $U \subset W$ be an open set, in the complex topology, with $U \cap S=\{0\}$, and let $V \subset X$ be such that $V=\pi^{-1}(U)$. We are in the hypothesis of Lemma 3.3; thus we get a "tautological" line bundle $H$ on $V$, and we conclude by [Fu1, Prop. $2.12]$.

There are two possible restrictions of $E$ to the fiber, namely, $E_{\mid F} \simeq \mathcal{O}(2) \oplus$ $\left(\oplus^{n-1} \mathcal{O}(1)\right)$ or $E_{\mid F}$ is the tangent bundle. As observed by Fujita in [Fu2, 3.8 and 3.11], these two restrictions have a different behavior in the diagram 2.1): in the former $\varphi$ is birational and $\operatorname{dim} F(\varphi)=n-2$, while in the latter it is of fiber type and $\operatorname{dim} F(\varphi)=n-3$. Hence the restriction has to be constant along all the fibers. 
Case $5.9(\operatorname{dim} W=3)$. The general fiber is $\mathbf{P}^{n-3}$ (see for instance [Fu2]). Assume that $n \geq 5$; therefore $\pi$ is elementary.

Since $\pi$ is elementary, any fiber $G$ has $\operatorname{cod} G \geq 2$. Let $S \subset W$ be the locus of point over which the fiber is not $\mathbf{P}^{n-3} ; \operatorname{dim} S \leq 0$ since a generic linear space section cannot intersect $S$, as above. Let $(W, 0)$ be an analytic germ of a smooth point of $W$. Then we are in the hypothesis of Proposition 2.5 and can assume that the contraction is supported (locally) by $K_{X}+(n-2) L$. Therefore, since $n \geq 5$, by $[\mathrm{AW}, \mathrm{Th} .4 .1]$ all the fibers have dimension $n-3$. We conclude that all fibers over $(W, 0)$ are $\mathbf{P}^{n-3}$.

Proof of part 3) of the theorem. In the last part of the theorem we assume that $K_{X}+\operatorname{det} E$ is nef and big but not ample. Then $K_{X}+\operatorname{det} E$ is a supporting divisor of an extremal face, $F$; let $R_{i}$ be the extremal rays spanning this face. Fix one of these rays, say $R=R_{i}$, and let $\pi: X \rightarrow W$ be the elementary contraction associated to $R$.

We have $l(R) \geq n-2$; this implies first that the exceptional loci are disjoint if $n>3$, by Proposition 1.11. Secondly, by the inequality 1.8), we have

$$
\operatorname{dim} E(R)+\operatorname{dim} F(\pi) \geq 2 n-3 .
$$

Therefore $\operatorname{dim} E(R)=n-1$ and either $\operatorname{dim} F(\pi)=n-1$ or $\operatorname{dim} F(\pi)=n-2$; thus $n-1 \geq l(R) \geq n-2$. If $B:=\rho(E)$ and $D=E(R)$, this implies that $\operatorname{dim} B=0$ or 1.

If $\operatorname{dim} B=1$ then $\operatorname{dim} F(\pi)=n-2$ for all fibers (note that since the contraction $\pi$ is elementary there cannot be a fiber of dimension $n-1)$; thus we can apply Theorem 3.1 with $r=n-2$. This will give the case 3(iii) of the theorem.

Now let $\operatorname{dim} B=0$ and consider again the construction in section 2; in particular we refer to the diagram 2.1). Let $S$ be the extremal ray contracted by $\varphi$; note that $l(S) \geq n-2$ and that the inequality 1.8$)$ gives

$$
\operatorname{dim} E(S)+\operatorname{dim} F(\varphi) \geq 3 n-6
$$

in particular, since $\operatorname{dim} F(\varphi) \leq \operatorname{dim} F(\pi)$, we have two cases, namely $\operatorname{dim} E(S)=$ $2 n-5$ and $\operatorname{dim} F(\varphi)=n-1$, or $\operatorname{dim} E(S)=2 n-4$ and $\operatorname{dim} F(\varphi)=n-1$ or $n-2$.

The case in which $\operatorname{dim} E(S)=2 n-5$ will not occur. In fact, after "slicing", (see (2.4)), we would obtain a map $\varphi^{\prime}=\varphi_{\mid Z}$ which would be a small contraction supported by a divisor of the type $K_{Z}+(n-2) L$, but this is impossible by the classification of [Fu1, Th. 4] (see also $[\mathrm{An}]$ ).

Hence $\operatorname{dim} E(S)=2 n-4$; that is, also $\varphi$ is divisorial and $E(S) \cdot l_{p}=0$, where $l_{p}$ is a line in $F(p)$. In particular, $E(S)=p^{*} D$.

Suppose that the general fiber of $\varphi, F(\varphi)$, has dimension $n-2$. After slicing we obtain a map $\varphi^{\prime}=\varphi_{\mid Z}: Z \rightarrow T$ supported by $K_{Z}+(n-2) L$, where $L=\xi_{E \mid Z}$. This map contracts divisors $\bar{D}$ in $Z$ to curves; by ([Fu1, Th. 4]) we know that every fiber $F$ of this map is $\mathbf{P}^{n-2}$ and that $\bar{D}_{\mid F}=\mathcal{O}(-1)$ (actually this map is a blow-up of a smooth curve in a smooth variety). In particular there are curves in $Y$, call them $l$, such that $-E(S) \cdot l=1$. We will discuss this case in a while.

Assume now that the general fiber and therefore all have dimension $n-1$.

Lemma 5.10. Under these hypotheses, $l(R)=n-2$.

Let $C$ be a minimal curve in $R$ (see 1.6)), $\nu: \mathbf{P}^{1} \rightarrow C$ its normalization, $\tilde{\nu}$ : $Y_{C}=\mathbf{P}\left(\nu^{*} E_{\mid C}\right) \rightarrow Y$ the induced morphism and $\xi_{C}$ the tautological bundle of $Y_{C}$; note that $\tilde{\nu}^{*} \xi_{E}=\xi_{C}$. 
Let $g: Y_{C} \rightarrow F(\psi)_{w}$ be the morphism induced by $\varphi$ on $Y_{C}$ and

$$
Y_{C} \stackrel{\alpha}{\rightarrow} V_{1} \stackrel{\beta}{\rightarrow} F(\psi)_{w}
$$

its Stein factorization. Assume by contradiction that $l(R)=n-1$; then $\nu^{*}\left(E_{\mid C}\right)=$ $\mathcal{O}(2) \oplus \mathcal{O}(1)^{\oplus n-3}$, and so $Y_{C}$ has two contractions, the scroll structure and a blowdown to $\mathbf{P}^{n-2}$.

Let $\tilde{l}$ be a line contracted by the blow-down; then $\tilde{l}$ is contracted by $g$. In fact, by the projection formula $\xi_{E} \cdot \tilde{\nu}_{*} \tilde{l}=\xi_{C} \cdot \tilde{l}=1$. Thus by the commutativity of the diagram $\tilde{\nu}_{*} \tilde{l}$ is a minimal curve in $\mathrm{S}$.

Since $\alpha$ cannot contract all $Y_{C}$, then $\alpha$ is the blow-down. Since $\operatorname{dim} F(\pi)=$ $\operatorname{dim} F(\varphi)$ by hypothesis, then by Remark 2.3 all fibers $F(\psi)$ have dimension $n-3$. So we get the contradiction that $\beta: \mathbf{P}^{n-2} \rightarrow F(\psi)_{w}$ is a finite map between two varieties of different dimension.

Slicing, we obtain a map $\varphi^{\prime}=\varphi_{\mid Z}: Z \rightarrow T$ supported by $K_{Z}+(n-2) L$, where $L=\xi_{E \mid Z}$. This map contracts divisors $\bar{D}$ in $Z$ to points; by ([Fu1]) we know that these divisors are either $\mathbf{P}^{n-1}$ with normal bundle $\mathcal{O}(-2)$ or $\mathbf{Q}^{n-1} \subset \mathbf{P}^{n}$ with normal bundle $\mathcal{O}(-1)$. In the latter case we have as above that there are curves $l$ in $Y$ such that $-E(S) \cdot l=1$.

In these cases observe that $E(S)=p^{*}(D)$ and $K_{X}+(n-2)(-D)$ is a supporting divisor for $\pi$. Then by [Fu1] we conclude that $\left(D, D_{\mid D}\right)$ is one of the pair listed in the theorem, and the theory of uniform bundles makes it easy to recover $E_{\mid D}$ $([\mathrm{OSS}])$.

There remains the case in which $\varphi^{\prime}=\varphi_{\mid Z}: Z \rightarrow T$ contracts divisors $\bar{D}=\mathbf{P}^{n-1}$ with normal bundle $\mathcal{O}(-2)$ to points. We can apply Proposition 2.5 and show that the singularities of $W$ are the same as those of $T$. Then, as in ([Mo1]), this means that we can factor $\pi$ with the blow-up of the singular point. Let $X^{\prime}=B l_{w}(W)$; then we have a birational map $g: X \rightarrow X^{\prime}$. Note that $X^{\prime}$ is smooth and that $g$ is finite. Actually it is an isomorphism outside $D$, and cannot contract any curve of $D$. Assume to the contrary that $g$ contracts a curve $C^{\prime} \subset D$; let $N \in \operatorname{Pic}\left(X^{\prime}\right)$ be an ample divisor. Then we have $g^{*} N \cdot C^{\prime}=0$ while $g^{*} N \cdot C \neq 0$, contradiction. Thus by Zariski's main theorem $g$ is an isomorphism. This gives the case in 3i).

\section{REFERENCES}

[An] Andreatta, M., Contractions of Gorenstein polarized varieties with high nef value, Math. Ann. 300 (1994), 669-679. MR 96b:14007

[ABW1] Andreatta, M., Ballico, E., Wiśniewski, J.A., On contractions of smooth algebraic varieties, preprint UTM 344 (1991).

[ABW2] ㄴ. Vector bundles and adjunction, International Journal of Mathematics,3 (1992), 331-340. MR 93h:14031

[AW] Andreatta, M., Wiśniewski, J. A., A note on nonvanishing and applications, Duke Math.J. 72 (1993), 739-755. MR 95c:14007

[BS] Beltrametti, M., Sommese, A. J. On the adjunction theoretic classification of polarized varieties, J. Reine Angew. Math. 427 (1992), 157-192. MR 93d:14012

[Fu1] Fujita, T., On polarized manifolds whose adjoint bundles are not semipositive, in Algebraic Geometry, Sendai, Adv. Studies in Pure Math. 10, Kinokuniya-North-Holland 1987, 167-178. MR 89d:14006

[Fu2] - On adjoint bundles of ample vector bundles, in Proc. Alg. Geom. Conf. Bayreuth (1990), Lect. Notes Math., 1507, 105-112. MR 93j:14052

[Fu3] On Kodaira energy and reduction of polarized manifolds, Manuscr. Math 76 (1992), 59-84. MR 93i: 14032 
[Io] Ionescu, P. Generalized adjunction and applications, Math. Proc. Camb. Phil. Soc. 99 (1986), 457-472. MR 87e:14031

[KMM] Kawamata, Y., Matsuda, K., Matsuki, K., Introduction to the minimal model program, in Algebraic Geometry, Sendai, Adv. Studies in Pure Math. 10, Kinokuniya-North-Holland 1987, 283-360. MR 89e:14015

[Ma] Maeda, H. Nefness of adjoint bundles for ample vector bundles, Le Matematiche (Catania) 50 (1995), 73-82. CMP 96:08

[Mo] Mori, S. Projective manifolds with ample tangent bundles, Ann. of Math. 110 (1979), 593-606. MR 81j:14010

[Mo1] , Threefolds whose canonical bundles are not numerically effective, Ann. of Math. 116 (1982), 133-176. MR 84e:14032

[Mu Mukai, S. Problems on characterizations of complex projective space, Birational Geometry of Algebraic Varieties - Open Problems, Katata, Japan (1988), 57-60.

[OSS] Okonek, C., Schneider, M., Spindler, H. Vector bundles on complex projective spaces, Birkhäuser, 1980. MR 81b:14001

[PSW] Peternell, T., Szurek, M., Wiśniewski, J. A., Fano manifolds and vector bundles, Math.Ann. 294 (1992), 151-165. MR 93h:14030

[So] Sommese, A. J., On the adjunction theoretic structure of projective varieties, Complex Analysis and Algebraic Geometry, Proceedings Göttingen, 1985 (ed. H. Grauert), Lecture Notes in Math., 1194 (1986), 175-213. MR 87m:14049

[Wi1] Wiśniewski, J. A., On a conjecture of Mukai, Manuscr. Math. 68 (1990), 135-141. MR 91f: 14040

[Wi2] , Length of extremal rays and generalized adjunction, Math. Z. 200 (1989), 409427. MR 91e: 14032

[Wi3] - On contraction of extremal rays of Fano manifolds, J. Reine Angew. Math. 417 (1991), 141-157. MR 92d:14032

[YZ] Ye, Y. G., Zhang, Q., On ample vector bundles whose adjunction bundles are not numerically effective, Duke Math. Journal, 60 (1990), 671-687. MR 91g:14040

[Zh] Zhang, Q., A theorem on the adjoint system for vector bundles, Manuscripta Math. 70 (1991), 189-201. MR 92c:14009

[Zh2] Ample vector bundles on singular varieties, Math. Zeit. 220 (1995), 59-64. MR 96i: 14034

Dipartimento di Matematica,Universitá di Trento, 38050 Povo (TN), Italia

E-mail address: andreatt@science.unitn.it

Dipartimento di Matematica,Universitá di Trento, 38050 Povo (TN), Italia

E-mail address: mella@science.unitn.it 